\title{
Edge Plasmon Polaritons on a Half-Plane
}

\author{
A.A. Zabolotnykh* and V.A. Volkov \\ Kotelnikov Institute of Radio-engineering and Electronics of the RAS, \\ Mokhovaya 11-7, Moscow 125009, Russia \\ and \\ Moscow Institute of Physics and Technology, \\ Institutskii per. 9, Dolgoprudny, \\ Moscow region 141700 , Russia
}

(Dated: September 3, 2018)

\begin{abstract}
The effect of electromagnetic retardation on the spectrum of edge plasmons in a semi-infinite two-dimensional electron system is considered. The problem is reduced to complicated integral equations for the potentials, which are solved upon a major simplification of the kernel. The spatial distribution of the potentials, charges, and currents is analyzed. It is shown that edge plasmon polaritons in the high-conductivity two dimensional system are characterized by a high Q factor at all frequencies, including those lower than the inverse electron relaxation time $\tau^{-1}$.
\end{abstract}

\section{INTRODUCTION}

Plasmons in two-dimensional (2D) electron systems formed in semiconductor heterostructures have been investigated for almost half a century, beginning with the pioneering theoretical work [1] and first experimental studies [2 4].

In contrast to the three-dimensional case, the spectrum of 2D plasmons has no gap at zero wave vector [1, 5] and, for a clean system (i.e., for the electron relaxation time $\tau \rightarrow \infty)$, in the quasistatic approximation and in the long-wavelength limiting case can be written as $\omega_{0}(q)=$ $\sqrt{2 \pi n_{0} e^{2} q / \varkappa m}$, where $q$ is the magnitude of the plasmon wave vector, $n_{0}$ is the unperturbed $2 \mathrm{D}$ electron density in the system, $m$ is the effective mass of the electron, $e>0$ is the elementary charge, and $\varkappa$ is the background dielectric constant.

For a finite electron relaxation time $\tau$, the plasmon frequency becomes complex-valued, with its imaginary part describing the plasmon decay with time. In the above approximations, the dispersion relation can be written as

$$
\omega_{b u l k}(q)=\omega^{\prime}+i \omega^{\prime \prime}=\sqrt{\omega_{0}^{2}(q)-1 / 4 \tau^{2}}-i / 2 \tau .
$$

One can see that the plasmon has a high $Q$ factor (i.e., $\left.\omega^{\prime} \gtrsim \omega^{\prime \prime}\right)$ in the frequency range above approximately $1 / \tau$. At lower frequencies, the plasmon decays more rapidly than it oscillates.

However, the situation changes radically if electromagnetic retardation is taken into consideration [6, 7]. The properties of 2D plasmon polaritons, i.e., plasmons with electromagnetic retardation taken into account, in a 2D electron system with a finite relaxation time $\tau$, were analyzed in [7]. It was found that the spectrum depends considerably on the dimensionless conductivity $\tilde{\sigma}=2 \pi \sigma / c$, where $\sigma=e^{2} n_{0} \tau / m$ is the static conductivity of the $2 \mathrm{D}$ electron system (which has the speed dimensionality in
Gaussian units) and $c$ is the speed of light (for simplicity, we consider a $2 \mathrm{D}$ system in vacuum; i.e., $\varkappa=1$ ). If the conductivity of the $2 \mathrm{D}$ system is low $(\widetilde{\sigma}<1)$, plasmon polaritons have a low $\mathrm{Q}$ factor and their spectrum is qualitatively similar to the plasmon spectrum given by Eq. (1) in the sense that the condition $\omega^{\prime} \geq \omega^{\prime \prime}$ is only satisfied beginning with some finite frequency $\omega^{\prime}$ and wave vector $q$. However, if the conductivity of the $2 \mathrm{D}$ system is high $(\widetilde{\sigma}>1)$, the plasmon polariton spectrum changes significantly (see 7], Fig. 1). In such a system, plasmon polaritons have a high $\mathrm{Q}$ factor, i.e., $\omega^{\prime} \gtrsim \omega^{\prime \prime}$, for all values of the wave vector $q$ and frequency $\omega^{\prime}$, including frequencies $\omega^{\prime}<1 / \tau$.

It is known that edge plasmons traveling along the boundary of a $2 \mathrm{D}$ system can exist [8 13]. In the quasistatic limiting case, their dispersion relation $\omega_{\text {edge }}(q)$ is similar to that of bulk plasmons (plasmons in a system with no boundary), see Eq. (1):

$$
\omega_{\text {edge }}(q)=\sqrt{\alpha^{2} \omega_{0}^{2}(q)-1 / 4 \tau^{2}}-i / 2 \tau,
$$

where $q$ is the wave vector along the boundary and the constant $\alpha \approx 0.906$ according to the exact solution of the problem given in [10] and $\alpha=\sqrt{2 / 3} \approx 0.816$ according to the approximate solution given in [11]. For $\omega^{\prime} \tau<1$, edge plasmons are strongly damped, similarly to bulk plasmons in the quasistatic limiting case.

The importance of electromagnetic retardation effects was noted in experimental studies on 2D plasmons [1419]. We should also mention theoretical studies on the microwave response of antidot arrays [20] and stripeshaped 2D electron systems [21]. The spectrum of plasmon polaritons in a double-layer system with a finite $\tau$ was considered in [22].

The goal of this study is to analyze the impact of electromagnetic retardation on the spectrum of edge plasmons (i.e., edge plasmon polaritons) in the simplest semiinfinite $2 \mathrm{D}$ electron system, which is a half-plane. It is essential that we use the simplest approach, developed by 
Fetter 11] in his treatment of the edge plasmon spectrum disregarding retardation.

\section{MAIN EQUATIONS AND THE METHOD OF SOLUTION}

Let us consider a $2 \mathrm{D}$ electron system in vacuum $(\varkappa=$ 1) occupying the half-plane $x>0, z=0$, so that its boundary coincides with the $y$-axis. We assume that the electron density drops stepwise to zero for $x<0$.

We are going to determine the spectrum of edge plasmon polaritons in the long-wavelength limiting case $q \ll$ $k_{F}$ (where $\hbar k_{F}$ is the Fermi momentum), because retardation plays the most important role when the plasmon wavelength is on the order of the wavelength of light with the same frequency. We will use the classical equation for the average velocity of electrons (the Euler equation) and Maxwell's equations.

The equation for the average electron velocity $\mathbf{v}=$ $\left(v_{x}, v_{y}\right)$ can be written as follows (see, e.g., [11, 23]):

$$
\partial_{t} \mathbf{v}+\mathbf{v} / \tau=-s^{2} \nabla n / n_{0}-e \mathbf{E} / m,
$$

where $\mathbf{E}=-\nabla \varphi-\partial_{t} \mathbf{A} / c$ is the mean field induced by electrons, $\mathbf{A}=\left(A_{x}, A_{y}\right)$ is the vector potential in the plane of the $2 \mathrm{D}$ electron system, and $n$ is the perturbation of the electron density with respect to its equilibrium value. In the first term on the right-hand side of Eq. (3), which describes pressure, $s$ is about the Fermi velocity $v_{F} ; s^{2}=3 v_{F}^{2} / 4$ according to [23].

Maxwell's equations for the scalar potential $\varphi$ and vector potential $\mathbf{A}=\left(A_{x}, A_{y}\right)$ and $A_{z}$ in the Lorentz gauge have the form

$$
\begin{aligned}
& \left(\frac{1}{c^{2}} \frac{\partial^{2}}{\partial t^{2}}-\Delta\right)\left(\begin{array}{l}
\varphi \\
\mathbf{A} \\
A_{z}
\end{array}\right)=4 \pi\left(\begin{array}{l}
\rho \\
\mathbf{j} / c \\
0
\end{array}\right) \delta(z), \\
& \operatorname{div} \mathbf{A}+\partial_{z} A_{z}+\partial_{t} \varphi / c=0,
\end{aligned}
$$

where div $=\left(\partial_{x}, \partial_{y}\right), \rho=-e n$ is the electron-density perturbation, and $\mathbf{j}$ is the $2 \mathrm{D}$ current density in the $2 \mathrm{D}$ electron system. A consequence of Eqs. (4) is the continuity equation $\partial_{t} \rho+\operatorname{div} \mathbf{j}=0$.

In the case of an unbounded system, Eqs. (3) and (4) define the spectra of independent TE and TM modes [7]. For large wave vectors (away from the light cone), the TM mode represents the conventional longitudinal plasmon with the spectrum given by Eq. (11). The existence of a boundary leads to the mixing of TE and TM modes.

Let us seek a solution in the form of a wave traveling along the boundary: $\mathbf{A}=\mathbf{A}(x, z) \exp \left(i q_{y} y-i \omega t\right)$ and $\varphi=\varphi(x, z) \exp \left(i q_{y} y-i \omega t\right)$, where $\mathbf{A}(x, z)$ and $\varphi(x, z)$ decrease away from the boundary. The vector potential component $A_{z}$ equals zero, because current does not flow perpendicularly to the $2 \mathrm{D}$ electron system. The component $A_{y}$ can be excluded using the gauge condition.
Thus, we focus on the equations for $\varphi(x, z)$ and $A_{x}(x, z)$ :

$$
\left(\partial_{x}^{2}+\partial_{z}^{2}-\beta^{2}\right)\left(\begin{array}{l}
\varphi(x, z) \\
A_{x}(x, z)
\end{array}\right)=4 \pi e\left(\begin{array}{l}
n(x) \\
\frac{n_{0} v_{x}(x)}{c}
\end{array}\right) \delta(z),
$$

where we have linearized the current density $\mathbf{j}=-e n_{0} \mathbf{v}$, $\beta=\sqrt{q_{y}^{2}-\omega^{2} / c^{2}}$ with $\operatorname{Re} \beta>0$, and $v_{x}(x)=0$ and $n(x)=0$ for $x<0$.

Using the Green's functions technique, one can reduce the set of equations (5) in 2D space to a set of integral equations in one-dimensional (1D) space. One can try to solve the resulting set of equations by the Wiener-Hopf method (see, e.g., [10]). However, the solution obtained in this way (if an explicit solution can be derived at all) is very cumbersome and hard to analyze. Thus, the plasmon spectrum in finite systems is frequently obtained using approximate methods. One of these methods, which we use here, is the simplification of the kernel in the integral equation for $\varphi(x, z)$ (and $A_{x}(x, z)$ ).

Seemingly, the simplification of the kernel of the integral equation was used for the first time to calculate the dispersion relation of $2 \mathrm{D}$ plasmons in [1], where the spectrum of the edge plasmon and magnetoplasmon for a semi-infinite system with a straight boundary was determined in the quasistatic approximation. In the case of the edge plasmon (without an external magnetic field), the spectrum obtained is in good agreement with the one determined from the exact solution [10]; the only difference is in the value of the constant $\alpha$ (see Introduction). Thus, one can hope that this method will yield reasonable results for the spectrum of the edge plasmon polariton as well. We mention that this method was used to calculate the spectra of plasmons in a strip [24], edge magnetoplasmons at the boundary between two 2D layers 25], and edge plasmons in graphene 26] and topological systems [27, 28].

Let us describe the essence of the method. Considering the first equation of the set (5) (the second equation can be treated in a similar way) for $z=0$, i.e., within the plane of the $2 \mathrm{D}$ system, and let us transform it to the $1 \mathrm{D}$ integral equation

$$
\varphi(x, z=0)=-2 e \int_{-\infty}^{+\infty} K_{0}\left(\beta\left|x-x^{\prime}\right|\right) n\left(x^{\prime}\right) d x^{\prime} .
$$

Here, $K_{0}(x)$ is the zero-order modified Bessel function of the second kind; its asymptotic behavior is $K_{0}(x)=$ $\sqrt{2 /(\pi x)} \exp (-|x|)$ for $x \rightarrow \infty$ and $K_{0}(x)=\ln (2 / x)-\gamma$ for $x \rightarrow 0$, where $\gamma \approx 0.577$ is the Euler-Mascheroni constant. The method consists in the replacement of the kernel $K_{0}(x)$ with a simpler one $L_{0}(x)$ characterized by the same area under the curve and the second moment (for details, see [11] and references therein). It proves that $L_{0}(x)=\pi \exp (-\sqrt{2} \beta|x|) / \sqrt{2}$ can be taken as an approximation for the kernel $K_{0}(x)$. As far as $L_{0}(x)$ is the Green's function for the operator $\left(-\partial_{x}^{2}+2 \beta^{2}\right) /(2 \pi \beta)$, 
we find that, after replacing $K_{0}(x)$ with $L_{0}(x)$ in Eq. (6), the latter can be transformed to the following set of differential equations for $\varphi(x, 0)$ and $A_{x}(x, 0)$ :

$$
\left\{\begin{array}{l}
\left(\partial_{x}^{2}-2 \beta^{2}\right) \varphi(x, 0)=4 \pi \beta e n(x) \\
\left(\partial_{x}^{2}-2 \beta^{2}\right) A_{x}(x, 0)=4 \pi \beta e n_{0} v_{x}(x) / c,
\end{array}\right.
$$

where $n(x)$ and $v_{x}(x)$ are equal to zero for $x<0$ and are finite for $x>0$. Thus, instead of integral equations (5), we obtain differential equations (7).

The substitution of the density $n$ from the continuity equation and of the current $\mathbf{j}$ from Eq. (3) into Eq. (7) provides the following set of equations for $x>0$ :

$$
\left\{\begin{array}{l}
s^{2} \varphi^{\prime \prime \prime \prime}(x)+\left(\omega \widetilde{\omega}-2 \omega_{p}^{2}-2 \beta^{2} s^{2}-s^{2} q_{y}^{2}\right) \varphi^{\prime \prime}(x) \\
+2 \beta^{2}\left(\omega_{p}^{2}-\omega \widetilde{\omega}+s^{2} q_{y}^{2}\right) \varphi(x)=0 \\
A_{x}^{\prime \prime}(x)-2\left(\beta^{2}+\omega_{p}^{2} \omega /\left(\widetilde{\omega} c^{2}\right)\right) A_{x}(x)= \\
\left(2 \omega_{p}^{2} \varphi^{\prime}(x)+2 \beta^{2} s^{2} \varphi^{\prime}(x)-s^{2} \varphi^{\prime \prime \prime}(x)\right) /(-i \widetilde{\omega} c),
\end{array}\right.
$$

where $\widetilde{\omega}=\omega+i / \tau, \omega_{p}^{2}=2 \pi e^{2} n_{0} \beta / m$, and $\varphi(x)$ and $A_{x}(x)$ are taken at $z=0$. We note that the equation for $\varphi(x)$ does not include the vector potential components. For $x<0$, we evidently obtain the following simple set of equations:

$$
\left\{\begin{array}{l}
\left(\partial_{x}^{2}-2 \beta^{2}\right) \varphi(x)=0 \\
\left(\partial_{x}^{2}-2 \beta^{2}\right) A_{x}(x)=0 .
\end{array}\right.
$$

Let us discuss the boundary conditions for Eqs. (8) and (9). First, we seek solutions localized near the boundary, i.e., decreasing for $x \rightarrow \pm \infty$. Second, we assume that $\varphi(x)$ and $A_{x}(x)$ along with their first derivatives are continuous at $x=0$; this follows from Eq. (7) and the absence of $\delta$-like (or even more singular) distributions of charges and currents at the boundary [29]. Third, the current (or velocity) component perpendicular to the boundary has to vanish at the boundary: $v_{x}(x=0)=0$.

Now, we proceed to the solution of sets of equations (8) and (9). Solving first the equation for $\varphi(x)$, we obtain

$$
\begin{array}{cc}
\varphi(x)=\varphi_{0} e^{\sqrt{2} \beta x}, & x<0 ; \\
\varphi(x)=\varphi_{1} e^{-\lambda_{1} x}+\varphi_{2} e^{-\lambda_{2} x}, & x>0 ;
\end{array}
$$

where $\varphi_{0,1,2}$ are constants. Provided that $s$ and $q_{y}$ are small, i.e. $s / c \ll 1,\left|\omega_{p}^{2}-\omega \widetilde{\omega}\right| \gg s^{2} q_{y}^{2}$, and $\left|2 \omega_{p}^{2}-\omega \widetilde{\omega}\right| \gg$ $s^{2}\left|q_{y}^{2}+2 \beta^{2}\right|$, we obtain

$$
\lambda_{1}^{2}=2 \beta^{2} \frac{\omega_{p}^{2}-\omega \widetilde{\omega}}{2 \omega_{p}^{2}-\omega \widetilde{\omega}}, \quad \lambda_{2}^{2}=\frac{2 \omega_{p}^{2}-\omega \widetilde{\omega}}{s^{2}},
$$

where the sign of $\lambda_{1,2}$ is determined from the condition $\operatorname{Re} \lambda_{1,2}>0$.

Next, we substitute the solution obtained for $\varphi(x)$ at $x>0$ into the right-hand side of the second equation of the set (8). We obtain

$$
\begin{array}{cc}
A_{x}(x)=A_{0} e^{\sqrt{2} \beta x}, & x<0 ;(13) \\
A_{x}(x)=A_{1} e^{-\lambda_{1} x}+A_{2} e^{-\lambda_{2} x}+A_{3} e^{-\gamma x}, & x>0 ;(14)
\end{array}
$$

where $A_{0,1,2,3}$ are constants, $\gamma^{2}=2\left(\beta^{2}+\omega_{p}^{2} \omega /\left(\widetilde{\omega} c^{2}\right)\right)$, and $\operatorname{Re} \gamma>0$. Constants $A_{1,2}$ are unambiguously related to $\varphi_{1,2}$ :

$$
A_{1,2}=\frac{\varphi_{1,2} \lambda_{1,2}\left(2 \omega_{p}^{2}+2 \beta^{2} s^{2}-s^{2} \lambda_{1,2}^{2}\right)}{i \widetilde{\omega} c\left(\lambda_{1,2}^{2}-\gamma^{2}\right)} .
$$

We have obtained five unknown constants $\left(\varphi_{0,1,2}\right.$ and $A_{0,3}$ ) and five boundary conditions at $x=0$ (the continuity of $\varphi(x), A_{x}(x)$, and their first-order derivatives; and the vanishing of the current at the boundary). Substituting the expressions obtained for $\varphi(x)$ and $A_{x}(x)$ into the boundary conditions, one can find the dispersion relation. Under the condition that $\omega \widetilde{\omega}$ is of the same order of magnitude as $2 \omega_{p}^{2}-\omega \widetilde{\omega}$, along with the conditions used to simplify the expressions for $\lambda_{1,2}$, we obtain

$$
\omega \widetilde{\omega}=D\left(2 \omega_{p}^{2}(1-\delta)-\omega \widetilde{\omega}\right),
$$

where $D=\sqrt{\frac{\omega_{p}^{2}-\omega \widetilde{\omega}}{2 \omega_{p}^{2}-\omega \widetilde{\omega}}}, \delta=\frac{\sqrt{1+\left(\omega_{p}^{2} \omega\right) /\left(c^{2} \widetilde{\omega} \beta^{2}\right)}-1}{D+\sqrt{1+\left(\omega_{p}^{2} \omega\right) /\left(c^{2} \widetilde{\omega} \beta^{2}\right)}}$.

In the quasistatic limiting case $(c \rightarrow \infty)$, the coefficient $\delta$ tends to zero, and we obtain the dispersion relation found in [11]. However, it is insufficient to simply replace $q_{y}$ with $\sqrt{q_{y}^{2}-\omega^{2} / c^{2}}$ in the dispersion relation without retardation in order to obtain Eq. (16).

\section{ANALYSIS OF THE DISPERSION RELATION}

The dispersion relation represented by Eq. (16) makes it possible to determine the spectrum of edge plasmonpolaritons, shown in Figs. 1 and 2

Let us first consider the case $\widetilde{\sigma}<1$ (Fig. 1). In this case, at small wave vectors, plasmon polaritons are characterized by a pure relaxation spectrum; for $q_{y}=0$, $\omega \tau=-i(1-\tilde{\sigma})$ or $\omega=0$. For large wave vectors, we obtain the usual spectrum given by Eq. (2) with $\alpha=\sqrt{2 / 3}$.

As $\widetilde{\sigma}$ approaches unity from below, the pure relaxation region "contracts" and vanishes for $\widetilde{\sigma}=1$.

The typical spectra of edge and bulk plasmon polariton for $\widetilde{\sigma}>1$ are shown in Fig. 2, Let us recall the characteristics of bulk plasmon polaritons for $\widetilde{\sigma}>1$. The asymptotic behavior of its spectrum for $q \rightarrow 0$ can be written as $\omega=\widetilde{\sigma} c q / \sqrt{\widetilde{\sigma}^{2}-1}-i \tau\left(\widetilde{\sigma} c q /\left(\widetilde{\sigma}^{2}-1\right)\right)^{2}$. Thus, $\omega^{\prime} \propto q$, $\omega^{\prime \prime} \propto q^{2}$, and plasmon polaritons are high-Q excitations at small wave vectors. Turning now to the spectrum of edge plasmon polaritons, we see that the asymptotic behavior at $q \rightarrow 0$ is linear for both $\omega^{\prime}$ and $\omega^{\prime \prime}$, in contrast to the bulk case; i.e., $\omega=v q_{y}$, where $v$ is the complexvalued velocity. The $v(\widetilde{\sigma})$ dependence is plotted in Fig. 3. It exhibits the asymptotic behavior

$$
v / c=\left\{\begin{array}{l}
\sqrt[4]{3}(1-i) /\left(2 \sqrt[4]{\widetilde{\sigma}^{2}-1}\right), \quad \widetilde{\sigma} \rightarrow 1+0 \\
1-(1+i \sqrt{3}) /\left(4 \sqrt[3]{4} \widetilde{\sigma}^{2 / 3}\right), \quad \tilde{\sigma} \rightarrow \infty
\end{array}\right.
$$




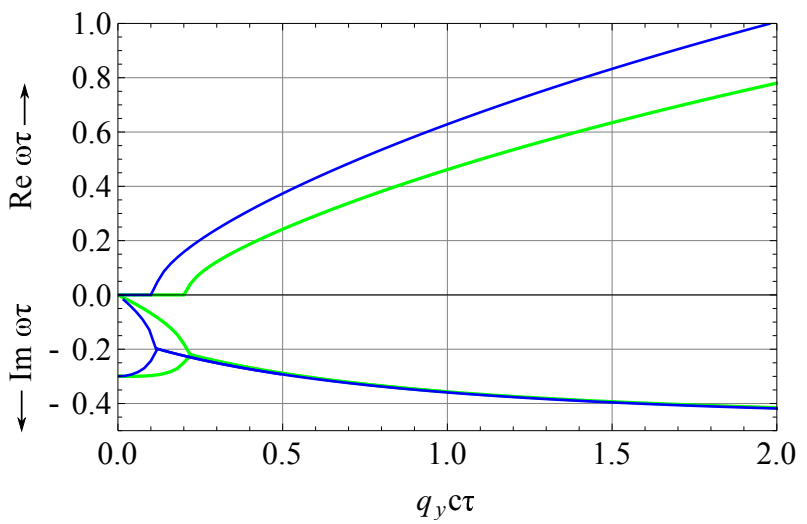

FIG. 1. Spectra of plasmon polaritons in a $2 \mathrm{D}$ system with the dimensionless conductivity $\tilde{\sigma}=2 \pi e^{2} n_{0} \tau /(m c)=0.7$. Green lines show the spectrum of the edge plasmon polariton; blue lines show the spectrum of the bulk plasmon polariton with the same value of $q_{y}$ and $q_{x}=0$. The real and imaginary parts of the complex-valued plasmon frequency, normalized to the electron relaxation time $\tau$, are given on the vertical axis in the top and bottom panels, respectively.

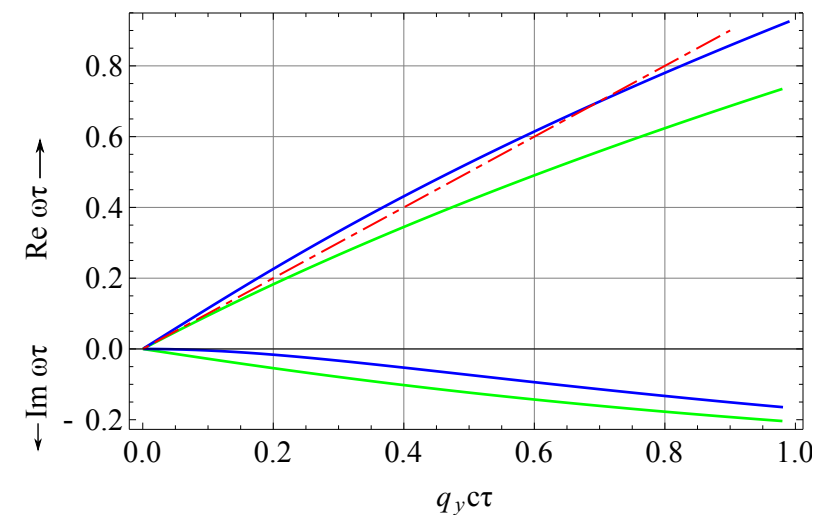

FIG. 2. Spectra of plasmon polaritons in a 2D system with the dimensionless conductivity $\widetilde{\sigma}=2$. Green lines show the spectrum of the edge plasmon polariton; blue lines show the spectrum of the bulk plasmon polariton with the same value of $q_{y}$ and $q_{x}=0$. The real and imaginary parts of the complexvalued plasmon frequency, normalized to the electron relaxation time $\tau$, are given on the vertical axis in the top and bottom panels, respectively. The red dash-dotted line shows the light cone $\left(\omega=c q_{y}\right)$.

The real part of the velocity Rev exceeds $c$ for $1<$ $\widetilde{\sigma}<\widetilde{\sigma}_{c}$, where $\widetilde{\sigma}_{c} \approx 1.57$ (see Fig. 3). For $\widetilde{\sigma} \rightarrow \infty$, the real part of the velocity $v$ approaches $c$ from below, while its imaginary part tends to zero, and $\operatorname{Re} v>\operatorname{Im} v$ for any $\tilde{\sigma}>1$. In other words, edge plasmon polaritons are "high-Q" excitations $\left(\omega^{\prime}>\omega^{\prime \prime}\right)$ for arbitrarily small values of $q_{y}$ and, consequently, for arbitrarily low frequencies $\omega^{\prime}$.

The characteristic localization length of the edge plasmon polariton field is determined by the root $\lambda_{1}$ and equals $1 / \operatorname{Re} \lambda_{1}$. For large values of $q_{y}$ (away from the

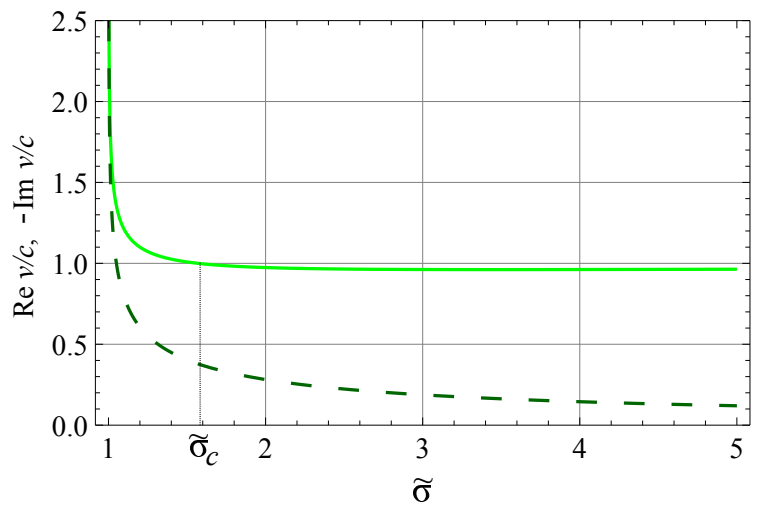

FIG. 3. (Solid line) Real part Rev and (dashed line) imaginary part $\operatorname{Im} v$ of the edge plasmon polariton velocity $v$ for $q_{y} \rightarrow 0$ versus the dimensionless conductivity $\widetilde{\sigma}>1$.

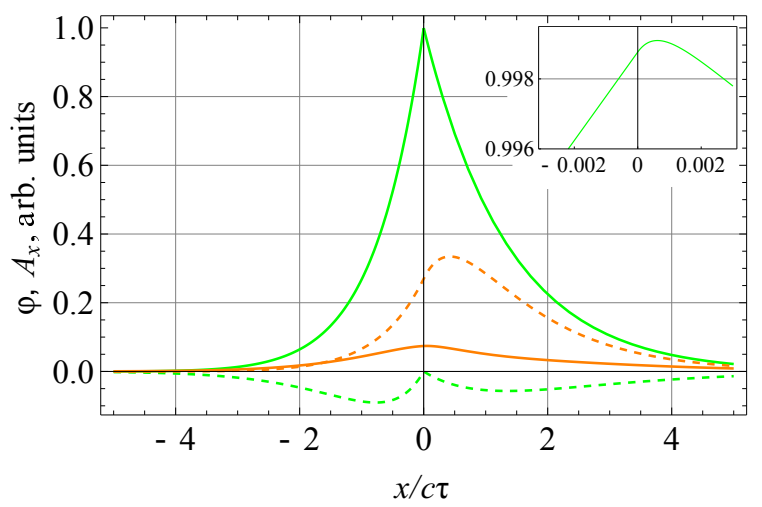

FIG. 4. Complex amplitudes of the scalar potential $\varphi(x, z=$ 0 ) and vector potential $A_{x}(x, z=0)$ (green and orange lines, respectively) in relative units (assuming $\varphi_{1}=1$ ) versus the coordinate $x$ normalized by $c \tau$; the $2 \mathrm{D}$ electron system occupies the region $x>0$. The solid and dashed lines show the real and imaginary parts of the potentials, respectively. The inset shows the part of the $\varphi$ dependence near zero on an expanded scale. The dependences are plotted for $\tilde{\sigma}=2$, $q_{y} c \tau=1.2$, and $\omega \tau \approx 0.86-i 0.23$ (see Fig. 2). For the ratio $c / s$, a typical value of $10^{3}$ was assumed. The characteristic localization length is $1 / \operatorname{Re} \lambda_{1} \approx 1.4 c \tau$.

light cone), $\operatorname{Re} \lambda_{1} \approx q_{y} / \sqrt{2}$. For $q_{y} \rightarrow 0$, we have $\lambda_{1}=q_{y}$ for $\widetilde{\sigma} \rightarrow 1+0$ and $\lambda_{1} \propto 1 / \sqrt[3]{\widetilde{\sigma}}$ for $\widetilde{\sigma} \rightarrow \infty$. Thus, for small $q_{y}$, the higher the conductivity, the larger the plasmon localization region. For large $q_{y}$, the localization region is independent of the conductivity and is of the order of $q_{y}^{-1}$. The typical dependences of the potentials $\varphi(x)$ and $A_{x}(x)$, as well as the charge density $\rho(x)$ and current density $j_{x}(x)$, on the coordinate $x$ are shown in Figs. 4 and 5. The parameters of the system are given in the caption of Fig. 4 ,

Let us discuss the spatial distribution of the charge. Some fraction of the charge is accumulated over a short length $1 / \operatorname{Re} \lambda_{2}$ (see Fig. 5 a , inset), while the rest of it is distributed over a longer length $1 / \operatorname{Re} \lambda_{1}$. We estimated the amount of charge concentrated at these two length 


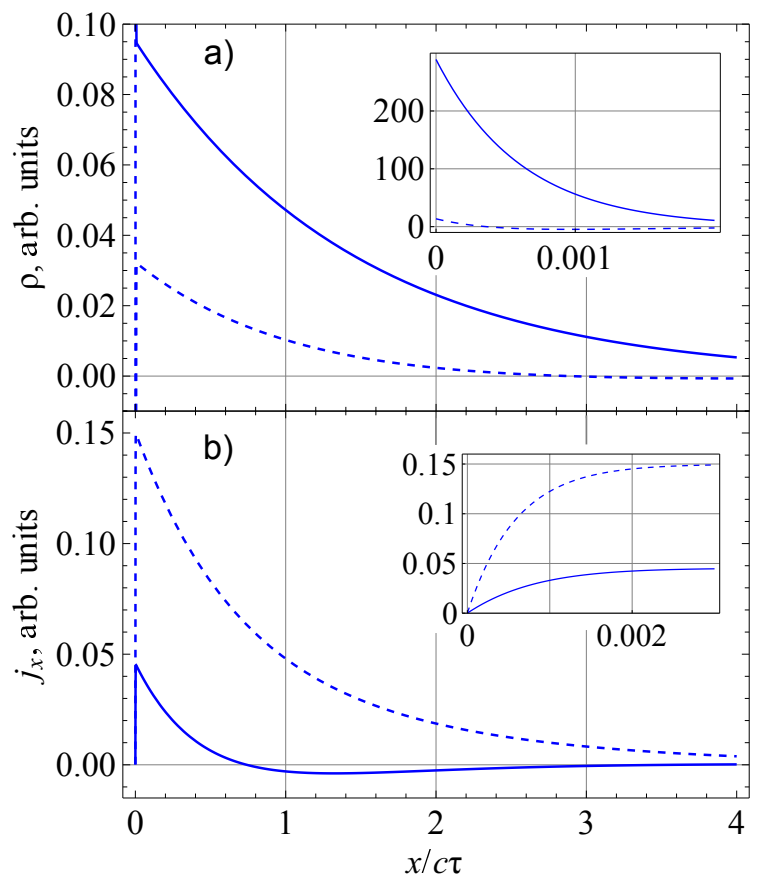

FIG. 5. Complex amplitudes of (a) the charge density $\rho(x)$ (normalized by $\varphi_{1} / c \tau$ ) and (b) the current density $j_{x}(x)$ (normalized by $\varphi_{1} / \tau$ ) versus the coordinate $x$ normalized by $c \tau$. The solid and dashed lines show the real and imaginary parts, respectively, of $\rho$ and $j_{x}$. The insets show the parts of the corresponding dependences near zero on an expanded scale. The dependences are plotted for the same parameters as in Fig. 4

scales. For large wave vectors $q_{y}$ (when the retardation effects are insignificant), approximately the same amount of charge is distributed at these two length scales. For $q_{y} \rightarrow 0$ and $\widetilde{\sigma} \rightarrow 1+0$, most of the charge is distributed at the longer length scale $1 / \operatorname{Re} \lambda_{1}$. For $q_{y} \rightarrow 0$ and $\widetilde{\sigma} \rightarrow \infty$, about $30 \%$ of all charge is distributed over the length $1 / \operatorname{Re} \lambda_{1}$.

\section{CONCLUSIONS}

To summarize, using the method developed by Fetter [11], we have found the approximate spectrum of edge plasmon polaritons in a semi-infinite $2 \mathrm{D}$ system with straight boundary. We have analyzed the spatial distribution of the potentials, charges, and currents. We have shown that, under the condition $2 \pi \sigma>c$ (where $\sigma$ is the static conductivity of the 2D system), the edge plasmon polariton, like its bulk counterpart, can be a high-Q excitation (i.e., Re $\omega \gtrsim \operatorname{Im} \omega$ ) for all wave vectors and, thus, for all frequencies, including frequencies lower than $\tau^{-1}$, where $\tau$ is the electron relaxation time in the $2 \mathrm{D}$ system.

The work was partly supported by the Russian Foundation for Basic Research (Projects Nos. 14-02-01166 and 16-32-00526). A.A.Z. acknowledges the support of the Dynasty Foundation.

* andrey.zabolotnyh@phystech.edu

[1] F. Stern, Phys. Rev. Lett. 18, 546 (1967).

[2] C. C. Grimes and G. Adams, Phys. Rev. Lett. 36, 145 (1976).

[3] S. J. Allen, Jr., D. C. Tsui, and R. A. Logan, Phys. Rev. Lett. 38, 980 (1977).

[4] T. N. Theis, J. P. Kotthaus, and P. J. Stiles, Solid State Commun. 26, 603 (1978).

[5] A. V. Chaplik, Sov. Phys. JETP 35, 395 (1972).

[6] A. O. Govorov and A. V. Chaplik, Sov. Phys. JETP 68, 1143 (1989).

[7] V. I. Fal'ko and D. E. Khmel'nitskii, Sov. Phys. JETP 68, 1150 (1989).

[8] D. B. Mast, A.J. Dahm, and A. L. Fetter, Phys. Rev. Lett. 54, 1706 (1985).

[9] D. C. Glattli, E. Y. Andrei, G. Deville, J. Poitrenaud, and F. I. B. Williams, Phys. Rev. Lett. 54, 1710 (1985).

[10] V. A. Volkov and S. A. Mikhailov, JETP Lett. 42, 556 (1985).

[11] A. L. Fetter, Phys. Rev. B 32, 7676 (1985).

[12] V. A. Volkov and S. A. Mikhailov, Sov. Phys. JETP 67, 1639 (1988).

[13] V. A. Volkov, D. V. Galchenkov, L. A. Galchenkov, I. M. Grodnenskii, O.R. Matov, and S.A. Mikhailov, JETP Lett. 44, 655 (1986).

[14] I. V. Kukushkin, J. H. Smet, S. A. Mikhailov, D. V. Kulakovskii, K. von Klitzing, and W. Wegscheider, Phys. Rev. Lett. 90, 156801 (2003).

[15] I. V. Kukushkin, D. V. Kulakovskii, S. A. Mikhailov, Yu. Smet, and K. von Klitzing, JETP Lett. 77, 497 (2003).

[16] I. V. Kukushkin, V. M. Muravev, J. H. Smet, M. Hauser, W. Dietsche, and K. von Klitzing, Phys. Rev. B 73, 113310 (2006).

[17] V. M. Muravev, I. V. Andreev, I. V. Kukushkin, S. Schmult, and W. Dietsche, Phys. Rev. B 83, 075309 (2011).

[18] P. A. Gusikhin, V. M. Muravev, and I. V. Kukushkin, JETP Lett. 100, 648 (2015).

[19] V. M. Muravev, P. A. Gusikhin, I. V. Andreev, and I. V. Kukushkin, Phys. Rev. Lett. 114, 106805 (2015).

[20] S. A. Mikhailov, Phys. Rev. B 54, 10335 (1996).

[21] S. A. Mikhailov and N. A. Savostianova, Phys. Rev. B 71, 035320 (2005).

[22] A. V. Chaplik, JETP Lett. 101, 545 (2015).

[23] S. Rudin and M. Dyakonov, Phys. Rev. B 55, 4684 (1997).

[24] V. Cataudella and G. Iadonisi, Phys. Rev. B 35, 7443 (1987).

[25] S. A. Mikhailov, JETP Lett. 61, 418 (1995).

[26] W. Wang, J. M. Kinaret, and S. P. Apell, Phys. Rev. B 85, 235444 (2012).

[27] J. C. W. Song and M. S. Rudner, PNAS 113, 4658-4663 (2016).

[28] A. Kumar, A. Nemilentsau, K. H. Fung, G. Hanson, N. X. Fang, and T. Low, Phys. Rev. B 93, 041413(R) (2016).

[29] L. A. Vainshtein, Diffraction Theory and Factorization Method (Sov. Radio, Moscow, 1966), p. 12 [in Russian]. 